\title{
Genomic epidemiology of a protracted hospital outbreak caused by multidrug-resistant Acinetobacter baumannii in Birmingham, England
}

Mihail R Halachev ${ }^{1 \dagger}$, Jacqueline Z-M Chan ${ }^{2 \dagger}$, Chrystala I Constantinidou ${ }^{2}$, Nicola Cumley ${ }^{3}$, Craig Bradley ${ }^{3}$, Matthew Smith-Banks ${ }^{3}$, Beryl Oppenheim ${ }^{3}$ and Mark J Pallen ${ }^{2^{*}}$

\begin{abstract}
Background: Multidrug-resistant Acinetobacter baumannii commonly causes hospital outbreaks. However, within an outbreak, it can be difficult to identify the routes of cross-infection rapidly and accurately enough to inform infection control. Here, we describe a protracted hospital outbreak of multidrug-resistant A. baumannii, in which whole-genome sequencing (WGS) was used to obtain a high-resolution view of the relationships between isolates.

Methods: To delineate and investigate the outbreak, we attempted to genome-sequence 114 isolates that had been assigned to the A. baumannii complex by the Vitek2 system and obtained informative draft genome sequences from 102 of them. Genomes were mapped against an outbreak reference sequence to identify single nucleotide variants (SNVs).

Results: We found that the pulsotype 27 outbreak strain was distinct from all other genome-sequenced strains. Seventy-four isolates from 49 patients could be assigned to the pulsotype 27 outbreak on the basis of genomic similarity, while WGS allowed 18 isolates to be ruled out of the outbreak. Among the pulsotype 27 outbreak isolates, we identified 31 SNVs and seven major genotypic clusters. In two patients, we documented within-host diversity, including mixtures of unrelated strains and within-strain clouds of SNV diversity. By combining WGS and epidemiological data, we reconstructed potential transmission events that linked all but 10 of the patients and confirmed links between clinical and environmental isolates. Identification of a contaminated bed and a burns theatre as sources of transmission led to enhanced environmental decontamination procedures.

Conclusions: WGS is now poised to make an impact on hospital infection prevention and control, delivering cost-effective identification of routes of infection within a clinically relevant timeframe and allowing infection control teams to track, and even prevent, the spread of drug-resistant hospital pathogens.
\end{abstract}

\section{Background}

Acinetobacter baumannii is an important cause of nosocomial infection, particularly ventilator-associated pneumonia and bloodstream infections in critically ill patients, and has a tendency to cause hospital outbreaks [1,2]. Multidrug-resistant (MDR) and even pan-drug-resistant strains have been reported worldwide [3]. It has also emerged as a threat to casualties of the conflicts in Iraq and Afghanistan, with the secondary problem that strains

\footnotetext{
*Correspondence: m.pallen@warwick.ac.uk

${ }^{\dagger}$ Equal contributors

${ }^{2}$ Division of Microbiology and Infection, Warwick Medical School, University of Warwick, Warwick CV4 7AL, UK

Full list of author information is available at the end of the article
}

introduced to hospitals by military personnel can cause cross infection of staff and patients [4-9]. Although existing molecular typing methods play an important role in identifying outbreaks $[10,11]$, they lack the resolution necessary to identify chains and modes of transmission within outbreaks and so can provide only limited guidance to infection control teams on how best to control or terminate an outbreak.

Whole-genome sequencing (WGS) of bacterial isolates provides a promising new method for investigating the epidemiology of outbreaks, particularly when coupled to clinical locational and temporal data [12-17]. Here, we describe a protracted hospital outbreak which occurred in Birmingham, England between July 2011 and 
February 2013 and was caused by a strain of Acinetobacter baumannii belonging to pulse-field gel electrophoresis type (pulsotype) 27. During the outbreak, we used genome sequencing to obtain a high-resolution view of the relationships between isolates, allowing us to reconstruct chains of transmission, confirm or refute epidemiological hypotheses and to provide the infection control team with useful insights into the sources and routes of infection during this outbreak.

\section{Methods}

\section{Microbiological investigations}

Here, we report a routine and clinically indicated infection control investigation into an outbreak, with no experimentation on human subjects. No additional samples other than those that were clinically relevant were taken from patients and the use of genome sequencing falls under the remit of laboratory method development, which does not need ethical approval. Multidrug-resistant Acinetobacter (MDR-Aci) isolates were obtained from routine clinical samples through culture on blood agar, followed by single-colony isolation. Bacterial identification and antibiotic susceptibility testing were performed in the hospital microbiology laboratory on the Vitek 2 system according to the manufacturer's recommendations (bioMérieux, Basingstoke, UK) [18]. Multidrug resistance was defined as resistance to $\geq 3$ classes of antibiotics (quinolones, extended-spectrum cephalosporins, $\beta$-lactam/ $\beta$-lactamase inhibitor combinations, aminoglycosides and carbapenems).

All MDR-Aci isolates from the Queen Elizabeth Hospital Birmingham during the outbreak period (July 2011 to February 2013) were considered for inclusion in the study. During this period, 65 patients tested positive for MDR-Aci in the clinical laboratory. Patients were numbered consecutively, based on the date of first isolation of MDR-Aci. The initial MDR-Aci isolate from each patient was sent to the Laboratory of HealthCare Associated Infection in Colindale, London for speciation and typing by pulsed-field gel electrophoresis (PFGE) and other molecular methods [10]. When the reference laboratory finds that two or more isolates from the UK share a novel PFGE pattern, the isolates are assigned to a new numerical pulsotype, for example, pulsotype 27 or pulsotype 29 .

An attempt was made to propagate isolates from all MDR-Aci-positive patients for genomic analysis. However, isolates from three patients (patients 15, 28 and 38) were lost on sub-culture or contaminated, leaving us with 74 genome-sequenced pulsotype 27 isolates from 58 patients. To examine within-host diversity, multiple isolates were obtained from 13 patients from different body sites and/or at different times. In addition, 18 isolates from 15 patients that had been identified as A. baumannii complex by Vitek 2, but turned out not to belong to the outbreak, were subjected to genome analysis, as were 10 environmental isolates and four control strains, which had been subjected to prolonged subculture in the laboratory. We also genome-sequenced the first pulsotype 27 isolate from the UK (kindly supplied by Jane Turton at the Laboratory of HealthCare Associated Infection), which was recovered in 2006 from a patient that had recently undergone surgery in India.

\section{Genomic and epidemiological investigation}

Genomic DNA was extracted from 114 putative Acinetobacter isolates, applying Qiagen 100/G Genomic-tips to 5 to $10 \mathrm{~mL}$ of overnight culture. A barcoded fragment library was generated for each isolate using the Nextera Sample Preparation and Nextera Index Kits (Illumina), then sequenced on an Illumina MiSeq, using paired-end $(2 \times 151$ or $2 \times 251)$ protocols, to give a minimum depth of coverage of $10 \times$. We implemented a filtering pipeline that trimmed reads at both ends, removing adaptors and bases with sequencing quality $<20$, and discarded all reads that mapped to PhiX or that contained Ns or where $>20 \%$ of bases had a sequencing quality of $<20$.

The genome of an isolate from a patient early in the outbreak (patient 6) was sequenced on two different sequencing platforms (454 FLX + and Illumina MiSeq), then a hybrid assembly was created to provide a reference genome for the outbreak, using Newbler v2.6 [19]. This assembly consisted of 4,031,405 base pairs, with 160 contigs in total and 126 contigs $>500 \mathrm{bp}$, with an N50 for contigs $>500$ base pairs of 31,936 base pairs. Five contigs (seq23, 67, 75, 100 and 128), comprising 77,648 base pairs/80 CDSs, were assigned to a cryptic plasmid on the basis of read depth, patterns of absence in some isolates and homology searches.

The outbreak reference genome was compared to all the MDR-Aci genome sequences that were publically available in May 2013, using the Average Nucleotide Identity (ANI) approach to identify the closest genomesequenced strain $[20,21]$. Isolates were assigned to a species on the basis of ANI to reference genomes [20,21]. For genotypic investigations of potential outbreaks, genome sequences were mapped to the relevant reference genome using Bowtie 2 [22], with default parameters, except that the reads were soft-clipped at the ends to improve the alignment score (option -local).

\section{SNV discovery procedure}

After mapping each set of read data to the reference genome as explained above, we processed with SAMtools v0.1.18 [23] (mpileup with default parameters, disabling the probabilistic realignment for the computation of base alignment quality, that is, we used option -B) and filtered it using BCFtools v0.1.17-dev (using the 
vcfutils.pl varFilter script to find variants with minimum root-mean-square mapping quality of 30 , maximum read depth of 10,000 and minimum distance to a gap of $150 \mathrm{bp}$, that is, approximately one read length). Using custom scripts, we screened these SNV locations to exclude some potentially spurious SNVs by retaining only SNVs which are:

- not from SNV-dense regions - no more than three SNVs in a 1,001 bp window centred on the SNV location

- most likely not from repeat regions - coverage less than twice the average isolate's coverage and

- at least $150 \mathrm{bp}$ from scaffold boundaries.

The alignments of the remaining variant loci were then manually inspected to check quality. For all SNV loci with coverage five-fold or less or with consensus $<90 \%$, we performed PCR and Sanger sequencing as a SNV verification step.

For isolates from the pulsotype 27 outbreak, we defined a new major SNV genotype, with a numerical designation (1.0, 2.0 and so on) whenever isolates with the same SNV profile were obtained from more than one patient. Genotypes confined to single patients within the outbreak were defined as minor genotypes and were given serial numerical designations (1.1, 2.1, 2.2 and so on) based on the closest relationship to a major genotype. All sequences from this study are available under ENA Accession number PRJEB4735.

To reconstruct the most parsimonious set of transmission events, we used a custom-built algorithm and script. We considered three modes of transmission: direct ward contact, where donor and recipient are on the same ward at the same time; delayed ward contact, where donor and recipient have been on the same ward but not at the same time; and theatre contact, where donor and recipient have received treatment in the same operating theatre. In the latter two modes, we assumed that MDR-Aci strains could survive in the hospital environment for up to 5 weeks [24].

\section{Results}

\section{Description of the Acinetobacter baumannii pulsotype 27} outbreak and the outbreak strain

Queen Elizabeth Hospital Birmingham (QEHB) is a public hospital with around 1,200 beds. British military casualties are usually repatriated here. In July 2011, we saw the first case in a new MDR-Aci outbreak: a military patient, who had been admitted to the hospital after suffering a blast injury in Afghanistan. The outbreak strain was identified in the clinical laboratory as an MDR-Aci showing resistance to multiple classes of antimicrobial agent, including aminoglycosides, fluoroquinolones, carbapenems, cephalosporins, carboxypenicillins and tetracycline. It was variably sensitive to colistin. The reference laboratory identified it as A. baumannii and, using PFGE, assigned it to pulsotype 27, a pulsotype not previously seen at QEHB.

Over the following 80 weeks, isolates of the outbreak strain were recovered from an additional 51 patients, including civilians and military casualties, spanning multiple wards (Figure 1). Isolates were assigned to the outbreak provisionally on the basis of antibiogram and definitively on the basis of pulsotype. However, after we began to apply WGS to putative MDR-Aci isolates in week 40 of the outbreak (April 2012), we also used genome sequence information to determine which isolates belonged to the outbreak. Analysis of a reference genome for the outbreak, created from an early outbreak isolate (isolate 6), revealed that the outbreak strain was distinct from all other well-characterised strains, including strains from previous outbreaks in local hospitals and clusters with other strains in a previously defined, widespread clonal lineage, International Clone I. A comparison with the first British pulsotype 27 isolate, which had been identified by the Laboratory of HealthCare Associated Infection in 2006, showed over 1,200 SNVs, ruling out any close relationship between our 2011 to 2013 outbreak and this historical isolate.

To delineate and investigate the outbreak, we attempted to genome-sequence 114 isolates identified as $A$. baumannii complex by the Vitek 2 system and obtained informative draft genome sequences from 102 of them. Seventy-four clinical isolates, obtained from 52 patients (including the index case), and 10 environmental isolates showed sufficient genomic similarity to the outbreak reference strain ( $\leq 8 \mathrm{SNVs}$ different) to be ruled into the outbreak (Table 1$)$.

\section{Genomics reveals clusters of infection}

We identified 31 SNVs in outbreak isolates (Table 2). These SNVs, together with presence/absence of a cryptic plasmid (that is, a $77-\mathrm{kb}$ plasmid with no obvious phenotype), define seven major outbreak genotypes, which fall into a phylogenetic relationship consistent with the timeline of the outbreak (Figure 2). Most of the major genotypes in the outbreak are accompanied by a cloud of one or two SNV variants in a wheel-and-spokes configuration, so that in total there are 32 distinct genotypes of the outbreak strain (Table 1, Figure 2). Laboratory subculture controls, including two different colonies picked after four serial subcultures and a culture that had been subjected to seven freeze-thaw cycles, all had the same SNV genotype as a minimally passaged parent culture, suggesting that SNVs are not readily acquired in the laboratory.

Among the outbreak genomes, we found two proteincoding genes that contained more than one SNV. Four non-synonymous SNVs were identified in the same sensor 


\section{a}

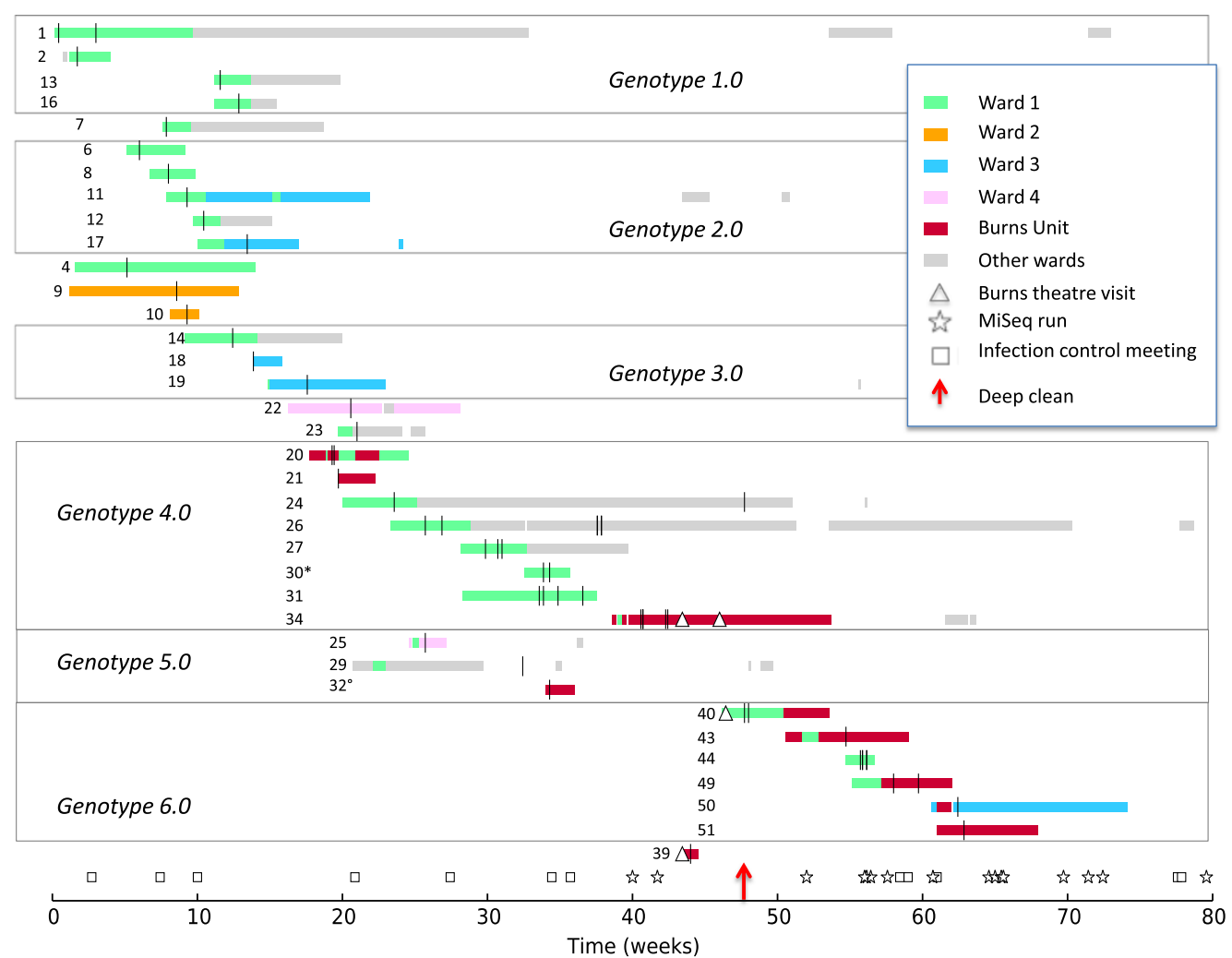

b

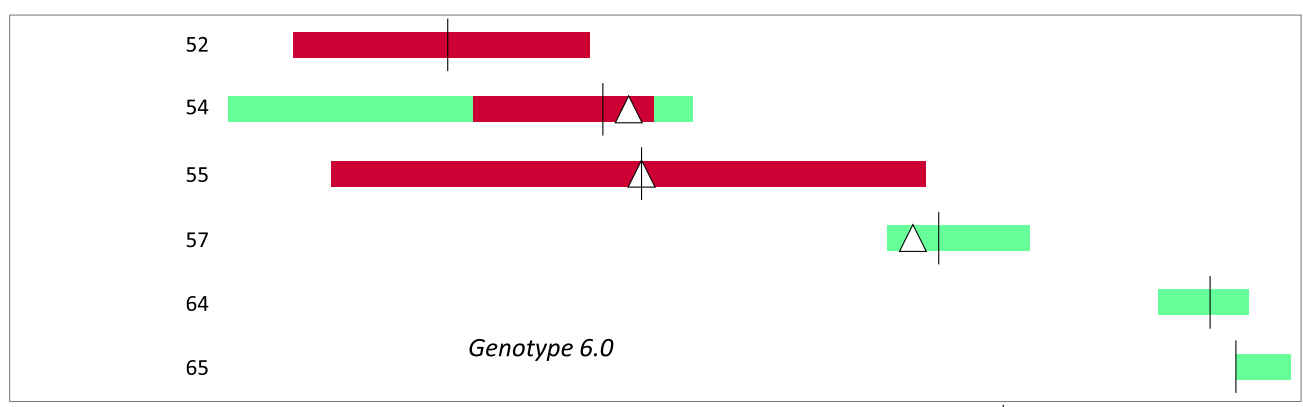

60

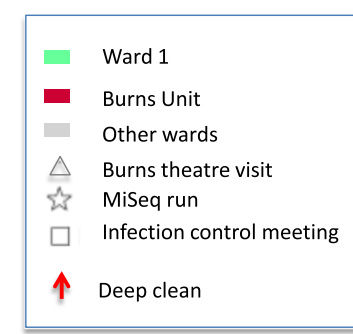

is
53

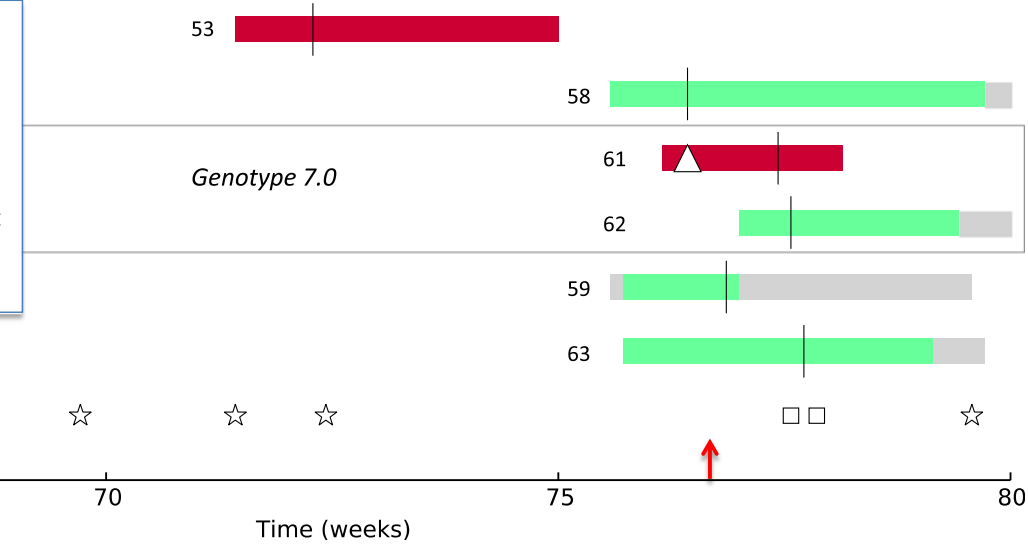

Figure 1 (See legend on next page.) 
(See figure on previous page.)

Figure 1 Chronology of the Acinetobacter baumannii pulsotype 27 outbreak in Birmingham, UK, 2011 to 2013 , showing ward occupancy and other events for $\mathbf{5 2}$ patients. (a) The first phase of the outbreak, up to week 70. (b) A detailed view of the second phase of the outbreak, after week 70. Vertical bars indicate samples positive for MDR-Aci. The coloured horizontal bars indicate ward occupancy by patients carrying MDR-Aci. Patients are ordered by the SNV genotype of their MDR-Aci isolates, with major genotypes delineated by rectangles. Ward 1 cares mainly for burns and trauma patients; Ward 2 cares mainly for cardiac surgery patients, Ward 3 cares mainly for trauma patients; Ward 4 for plastic, ear-nose-and-throat, maxillofacial, trauma patients. ${ }^{*}$ The first of three isolates obtained from patient 30 was not genome-sequenced.

- Patient 32 visited Ward 1 for 12 hours.

kinase gene, $p m r B$, which has been implicated in colistin resistance [25]. None of these SNVs match known colistin resistance-associated mutations. However, three of the changes occur in isolates (1b, 34e and 23) that show decreased susceptibility to colistin (MIC $256 \mathrm{mg} / \mathrm{L}$ ), suggesting that they might represent new resistance-associated mutations. Isolates that share the fourth SNV in $p m r B$, which delineates patient 22 from Genotype 2.0 and also occurs in Genotypes 3.0-7.0, retain sensitivity to colistin $(\mathrm{MIC} \leq 8 \mathrm{mg} / \mathrm{L})$.

\section{Non-outbreak isolates, within-host diversity and mixed infections}

Genome sequencing revealed that 18 Acinetobacter isolates, although obtained from hospitalised patients during the study period, did not belong to the main outbreak. A close genomic relationship between four isolates, twinned with a 3-week overlap in ward occupancy, provided evidence of a second small outbreak of MDRAci, which from PFGE results on three of the isolates could be assigned to pulsotype 29 (Table 3). Similarly, genome comparisons established that seven isolates obtained from five patients were members of the related species, Acinetobacter pittii, but very large pair-wise differences (>20,000 SNVs) between $A$. pittii isolates from different patients ruled out cross-infection.

From one trauma patient (patient 26), who was hospitalised for over 7 months, we genome-sequenced seven isolates of MDR-Aci obtained from different anatomical sites over a 4-month period and found five SNV variants (Figure 2):

- The initial isolate, 26a, which was obtained from a sputum sample, falls one SNV away from Genotype 4.0.

- A blood isolate (26b) taken 8 days later falls within Genotype 4.0.

- Isolates $26 \mathrm{c} / \mathrm{d} / \mathrm{f}$, obtained from a series of CSF samples taken approximately 3 months later, fall one SNV away from 26a

- A second sputum isolate (26e) represents a unique one-SNV variant of genotype 4.0.

Retrieval of a cloud of genotypes from a single patient illustrates the potential for within-host evolution in
MDR-Aci, mirroring findings with other hospital pathogens such as Staphylococcus aureus [26,27].

From yet another CSF sample from patient 26, we isolated a strain of MDR-Aci that was shown to be distinct from the outbreak strain by PFGE typing and by genome sequencing, providing evidence of double infection. We also found evidence of double infection with Acinetobacter in another trauma patient, patient 44, where two isolates, each from a separate wound swab taken on the same day, were identified by genome sequencing as $A$. pittii and the outbreak strain of A. baumannii.

\section{Routes and chains of transmission within the main MDR-Aci outbreak}

We reconstructed transmission events, assuming the most parsimonious transmission paths between patients. Using conventional epidemiological information alone, we identified 273 potential transmission events - an average of approximately five per patient - that might link patients within the outbreak. When genome sequence data were included, we were able to reduce this to a set of 57 potential transmission events. This set linked all but 10 of the pulsotype 27 patients and, in most cases, provided a single most-parsimonious transmission event that explained how a patient acquired the outbreak strain (Table 4).

Early in the outbreak, epidemiological and genomic analyses indicated that transmission occurred primarily as a result of cross-infection between patients located on the same ward at the same time. Thus, all isolates from Genotypes 1.0 and 2.0 and most of the isolates from Genotype 4.0 came from patients who had stayed on the Ward 1. In some cases, long-term contamination of the ward environment was thought to account for transmission and this was confirmed by environmental swabbing in side rooms after patients had been discharged and the room cleaned (Table 1). For example, isolate E1 was recovered a day after patient 44 was discharged; genomic analyses revealed it shared the same SNV profile (Genotype 6.0) as four of the five MDR-Aci isolates from that patient. Similarly isolates E2-4 were taken a day after patient 55 was discharged and were found to show a oneSNV difference from a patient 55 isolate. In both cases, the patients suffered severe burns and each stayed in a single room for the entire hospital stay. Confirmation of 
Table 1 Description of 52 patients and 84 isolates associated with the Acinetobacter baumannii pulsotype 27 outbreak in Birmingham, England, 2011 to 2013

\begin{tabular}{|c|c|c|c|c|c|c|}
\hline \multirow{2}{*}{$\begin{array}{l}\text { Patient no. or } \\
\text { environmental source }\end{array}$} & \multirow{2}{*}{$\begin{array}{l}\text { Length of hospital } \\
\text { stay (days) }\end{array}$} & \multirow{2}{*}{$\begin{array}{l}\text { Isolate } \\
\text { no. }\end{array}$} & \multicolumn{2}{|c|}{ Time of isolation (days) } & \multirow[t]{2}{*}{ Genotype } & \multirow{2}{*}{$\begin{array}{l}\text { SNVs/plasmid loss } \\
\text { ( } p \text { indicates loss of plasmid }\end{array}$} \\
\hline & & & From admission & $\begin{array}{l}\text { From start of } \\
\text { outbreak }\end{array}$ & & \\
\hline \multirow[t]{2}{*}{1} & \multirow[t]{2}{*}{231} & $1 \mathrm{a}$ & 3 & 3 & 1.0 & 0 \\
\hline & & $1 b$ & 21 & 21 & 1.1 & $1, p$ \\
\hline 2 & 24 & 2 & 7 & 12 & 1.0 & 0 \\
\hline 4 & 88 & 4 & 25 & 36 & 2.1 & $2,4, p$ \\
\hline 6 & 29 & 6 & 6 & 42 & 2.0 & 2 \\
\hline 7 & 422 & 7 & 3 & 55 & 1.2 & 3 \\
\hline 8 & 23 & 8 & 9 & 56 & 2.0 & 2 \\
\hline 9 & 83 & 9 & 52 & 60 & 2.2 & 2,7 \\
\hline 10 & 15 & 10 & 65 & 65 & 2.3 & $2,7,10,11$ \\
\hline 11 & 99 & 11 & 11 & 65 & 2.0 & 2 \\
\hline 12 & 39 & 12 & 6 & 73 & 2.0 & 2 \\
\hline 13 & 62 & 13 & 3 & 81 & 1.0 & 0 \\
\hline 14 & 77 & 14 & 24 & 87 & 3.0 & 2,6 \\
\hline 15 & \multicolumn{6}{|c|}{ LOST: not included in transmission analysis } \\
\hline 16 & 31 & 16 & 12 & 90 & 1.0 & 0 \\
\hline 17 & 535 & 17 & 24 & 94 & 2.0 & 2 \\
\hline 18 & 15 & 18 & 2 & 97 & 3.0 & 2,6 \\
\hline 19 & 58 & 19 & 19 & 123 & 3.0 & 2,6 \\
\hline \multirow[t]{2}{*}{20} & \multirow[t]{2}{*}{49} & $20 a$ & 12 & 135 & 4.0 & $2,5,9$ \\
\hline & & $20 b$ & 13 & 136 & 4.0 & $2,5,9$ \\
\hline 21 & 19 & 21 & 1 & 138 & 4.0 & $2,5,9$ \\
\hline 22 & 84 & 22 & 31 & 144 & 2.4 & 2,5 \\
\hline 23 & 45 & 23 & 10 & 147 & 2.5 & $2,5,8$ \\
\hline \multirow[t]{2}{*}{24} & \multirow[t]{2}{*}{218} & $24 a$ & 25 & 165 & 4.0 & $2,5,9$ \\
\hline & & $24 b$ & 194 & 334 & 4.10 & $2,5,9,12, p$ \\
\hline 25 & 19 & 25 & 8 & 180 & 5.0 & $2,5,9, p$ \\
\hline \multirow[t]{6}{*}{26} & \multirow[t]{6}{*}{197} & $26 a$ & 17 & 180 & 4.1 & $2,5,9,13$ \\
\hline & & $26 b$ & 25 & 188 & 4.0 & $2,5,9$ \\
\hline & & $26 c$ & 100 & 263 & 4.2 & $2,5,9,13,22, p$ \\
\hline & & $26 d$ & 100 & 263 & 4.2 & $2,5,9,13,22, p$ \\
\hline & & $26 e$ & 102 & 265 & 4.6 & $2,5,9,14$ \\
\hline & & $26 f$ & 102 & 265 & 4.2 & $2,5,9,13,22, p$ \\
\hline \multirow[t]{3}{*}{27} & \multirow[t]{3}{*}{82} & $27 a$ & 13 & 209 & 4.0 & $2,5,9$ \\
\hline & & $27 b$ & 19 & 215 & 4.3 & $2,5,9,15$ \\
\hline & & $27 c$ & 21 & 217 & 4.0 & $2,5,9$ \\
\hline \multirow[t]{3}{*}{28} & \multirow[t]{3}{*}{114} & $28 a$ & 31 & 227 & \multirow{3}{*}{\multicolumn{2}{|c|}{ LOST: mixed culture }} \\
\hline & & $28 b$ & 43 & 239 & & \\
\hline & & $28 \mathrm{c}$ & 61 & 257 & & \\
\hline 29 & 64 & $29^{*}$ & 83 & 227 (GP) & 5.0 & $2,5,9, p$ \\
\hline \multirow[t]{2}{*}{30} & \multirow[t]{2}{*}{23} & $30 a$ & 10 & 237 & 4.0 & $2,5,9$ \\
\hline & & $30 b$ & 13 & 240 & 4.0 & $2,5,9$ \\
\hline
\end{tabular}


Table 1 Description of 52 patients and 84 isolates associated with the Acinetobacter baumannii pulsotype 27 outbreak in Birmingham, England, 2011 to 2013 (Continued)

\begin{tabular}{|c|c|c|c|c|c|c|}
\hline \multirow{2}{*}{$\begin{array}{l}\text { Patient no. or } \\
\text { environmental source }\end{array}$} & \multirow{2}{*}{$\begin{array}{l}\text { Length of hospital } \\
\text { stay (days) }\end{array}$} & \multirow{2}{*}{$\begin{array}{l}\text { Isolate } \\
\text { no. }\end{array}$} & \multicolumn{2}{|c|}{ Time of isolation (days) } & \multirow[t]{2}{*}{ Genotype } & \multirow{2}{*}{$\begin{array}{l}\text { SNVs/plasmid loss } \\
\text { (p indicates loss of plasmid }\end{array}$} \\
\hline & & & From admission & $\begin{array}{l}\text { From start of } \\
\text { outbreak }\end{array}$ & & \\
\hline \multirow[t]{11}{*}{31} & 66 & $31 a$ & 37 & 235 & 4.0 & $2,5,9$ \\
\hline & & $31 b$ & 37 & 235 & MIXED & \\
\hline & & $31 c$ & 39 & 237 & 4.4 & $2,5,9,18$ \\
\hline & & $31 d$ & 39 & 237 & \multicolumn{2}{|c|}{ LOST: mixed culture } \\
\hline & & $31 e$ & 39 & 237 & \multicolumn{2}{|c|}{ Escherichia coli } \\
\hline & & $31 f$ & 39 & 237 & \multicolumn{2}{|c|}{ LOST: mixed culture } \\
\hline & & $31 \mathrm{~g}$ & 39 & 237 & \multicolumn{2}{|c|}{ LOST: mixed culture } \\
\hline & & $31 \mathrm{~h}$ & 39 & 237 & \multicolumn{2}{|c|}{ LOST: mixed culture } \\
\hline & & $31 i$ & 46 & 244 & 4.5 & $2,5,9,17$ \\
\hline & & $31 j$ & 58 & 256 & 4.0 & $2,5,9$ \\
\hline & & $31 \mathrm{k}$ & 64 & 297 & \multicolumn{2}{|c|}{ Pseudomonas aeruginosa } \\
\hline 32 & 16 & 32 & 4 & 240 & 5.0 & $2,5,9, p$ \\
\hline \multirow[t]{6}{*}{34} & 107 & $34 a$ & 14 & 284 & 4.7 & $2,5,9,19$ \\
\hline & & $34 b$ & 15 & 285 & 4.0 & $2,5,9$ \\
\hline & & $34 c$ & 15 & 285 & 4.0 & $2,5,9$ \\
\hline & & $34 d$ & 15 & 285 & 4.0 & $2,5,9$ \\
\hline & & $34 \mathrm{e}$ & 26 & 296 & 4.8 & $2,5,9,20$ \\
\hline & & $34 f$ & 27 & 297 & 4.9 & $2,5,9,28$ \\
\hline 38 & 96 & 38 & 39 & 298 & \multicolumn{2}{|l|}{ LOST } \\
\hline 39 & 9 & 39 & 4 & 308 & 6.1 & $2,5,9,16,23$ \\
\hline \multirow[t]{2}{*}{40} & \multirow[t]{2}{*}{53} & $40 a$ & 11 & 334 & 6.0 & $2,5,9,16$ \\
\hline & & $40 \mathrm{~b}$ & 13 & 336 & 6.0 & $2,5,9,16$ \\
\hline 43 & 60 & 43 & 29 & 383 & 6.0 & $2,5,9,16$ \\
\hline \multirow[t]{5}{*}{44} & \multirow[t]{5}{*}{15} & $44 a$ & 8 & 390 & 6.0 & $2,5,9,16$ \\
\hline & & $44 b$ & 9 & 391 & 6.0 & $2,5,9,16$ \\
\hline & & $44 c$ & 11 & 391 & 6.2 & $2,5,9,16,24$ \\
\hline & & $44 e$ & 11 & 393 & 6.0 & $2,5,9,16$ \\
\hline & & $44 f$ & 11 & 393 & 6.0 & $2,5,9,16$ \\
\hline \multicolumn{2}{|l|}{ Ward 1 post-patient 44} & E1 & & 397 & 6.0 & $2,5,9,16$ \\
\hline \multirow[t]{2}{*}{49} & \multirow[t]{2}{*}{49} & $49 a$ & 21 & 406 & 6.0 & $2,5,9,16$ \\
\hline & & $49 b$ & 33 & 418 & 5.0 & $2,5,9, p$ \\
\hline 50 & 50 & 50 & 14 & 437 & 6.0 & $2,5,9,16$ \\
\hline 51 & 96 & 51 & 14 & 440 & 6.0 & $2,5,9,16$ \\
\hline 52 & 24 & 52 & 13 & 495 & 6.0 & $2,5,9,16$ \\
\hline 53 & 26 & 53 & 6 & 506 & 4.11 & $2,5,9,21, p$ \\
\hline 54 & 37 & 54 & 30 & 507 & 6.0 & $2,5,9,16$ \\
\hline 55 & 47 & 55 & 25 & 510 & 6.0 & $2,5,9,16$ \\
\hline \multicolumn{2}{|c|}{ Burns Unit shower head post-patient 55} & E2 & & 532 & 5.0 & $2,5,9, p$ \\
\hline \multicolumn{2}{|c|}{ Burns Unit shower chair post-patient 55} & E3 & & 532 & 5.0 & $2,5,9, p$ \\
\hline Burns Unit patient chair & -patient 55 & E4 & & 532 & 5.0 & $2,5,9, p$ \\
\hline 57 & 12 & 57 & 5 & 533 & 6.0 & $2,5,9,16$ \\
\hline
\end{tabular}


Table 1 Description of 52 patients and 84 isolates associated with the Acinetobacter baumannii pulsotype 27 outbreak in Birmingham, England, 2011 to 2013 (Continued)

\begin{tabular}{|c|c|c|c|c|c|c|}
\hline \multirow{2}{*}{$\begin{array}{l}\text { Patient no. or } \\
\text { environmental source }\end{array}$} & \multirow{2}{*}{$\begin{array}{l}\text { Length of hospital } \\
\text { stay (days) }\end{array}$} & \multirow{2}{*}{$\begin{array}{l}\text { Isolate } \\
\text { no. }\end{array}$} & \multicolumn{2}{|c|}{ Time of isolation (days) } & \multirow[t]{2}{*}{ Genotype } & \multirow{2}{*}{$\begin{array}{l}\text { SNVs/plasmid loss } \\
\text { (p indicates loss of plasmid) }\end{array}$} \\
\hline & & & From admission & $\begin{array}{l}\text { From start of } \\
\text { outbreak }\end{array}$ & & \\
\hline \multicolumn{2}{|c|}{ Touch screen burns theatre post-patient 57} & E5 & & 538 & 7.0 & $2,5,9,16,26,29$ \\
\hline \multicolumn{2}{|c|}{ Anaesthetic machine burns theatre post-patient 57} & E6 & & 538 & 7.0 & $2,5,9,16,26,29$ \\
\hline \multicolumn{2}{|c|}{ Pat Slide burns theatre post-patient 57} & E7 & & 538 & 6.4 & $2,5,9,16,26$ \\
\hline \multicolumn{2}{|c|}{ Stool burns theatre post-patient 57} & E8 & & 538 & 6.4 & $2,5,9,16,26$ \\
\hline \multicolumn{2}{|c|}{ Scissors burns theatre post-patient 57} & E9 & & 538 & 6.5 & $2,5,9,16,27$ \\
\hline \multicolumn{2}{|c|}{ ECG leads burns theatre post-patient 57} & E10 & & 538 & 7.0 & $2,5,9,16,26,29$ \\
\hline 58 & 72 & 58 & 6 & 535 & 6.3 & $2,5,9,16,25$ \\
\hline 59 & 29 & 59 & 9 & 538 & 7.1 & $2,5,9,16,26,29,30, p$ \\
\hline 60 & 36 & 60 & 19 & 538 & 5.0 & $2,5,9, p$ \\
\hline 61 & 15 & 61 & 9 & 542 & 7.0 & $2,5,9,16,26,29$ \\
\hline 62 & 27 & 62 & 4 & 543 & 7.0 & $2,5,9,16,26,29$ \\
\hline 63 & 29 & 63 & 15 & 544 & 7.2 & $2,5,9,16,26,29,31$ \\
\hline 64 & 8 & 64 & 4 & 554 & 6.0 & $2,5,9,16$ \\
\hline 65 & 15 & 65 & 2 & 556 & 6.0 & $2,5,9,16$ \\
\hline
\end{tabular}

Patients were assigned to the outbreak if an initial isolate was shown by PFGE to belong to pulsotype 27. For three patients (15, 28, 38), no MDR-Aci isolates were available for genome sequencing.

*Isolate 29 was obtained after discharge from hospital from a sample provided by a general practitioner (GP).

contamination of the hospital environment led to a tightening of ward decontamination procedures.

Some outbreak strain acquisitions could not be explained simply by within-ward transmission, so we were forced to consider alternative routes of infection. As the outbreak progressed, we noticed that most of the affected patients made numerous visits to operating theatres: only five were never treated in an operating theatre. One particular theatre, specializing in the treatment of burns patients, was implicated in transmission between patient 34 (donor) and patients 40 and 39 (recipients). Consequently, in week 46 the burns theatre was closed and underwent deep cleaning (that is, decluttering of the operating theatre, followed by cleaning of all patient-associated equipment, non-fixed items, horizontal surfaces, walls, ceilings, ventilation shafts and storage areas with a chlorine-based disinfectant). Although there were several ward-based transmission events in the weeks that followed, no new theatre-acquired cases were observed for the subsequent 6 weeks and, for a time, the outbreak appeared to have ended.

Unfortunately, the outbreak resumed when a burns patient, patient 52, presented with an isolate from Genotype 6.0 in week 70. Initial epidemiological investigations failed to find any plausible direct ward- or theatrebased route of transmission that might link patient 52 with earlier outbreak cases. However, our finding of genotypic identity between the patient 52 isolate and previous outbreak isolates forced us to perform a more thorough epidemiological investigation, which uncovered a vehicle for transmission: patient 52 had occupied a specialised burns care bed that had been previously occupied by another Genotype 6.0 patient, patient 50. This prompted the development of a decontamination protocol for this specialised type of bed.

The outbreak spread to over a dozen new patients during the subsequent 9 weeks. Our suspicion once again focused on the burns theatre as the likely source of infection. This was confirmed when we obtained six isolates (E5-10) from environmental swabs of the burns operating theatre. All isolates from this phase of the outbreak, from patients and the environment, belonged to, or were closely related to, Genotypes 6.0 and 7.0. These findings prompted a second closure of the burns theatre, with deep cleaning in week 76. Following this second deep clean of the theatre the outbreak ceased and no further acquisitions of the strain were identified. The outbreak was formally declared closed in May 2013 when no inpatients were colonised or infected with the outbreak strain and there had been no new acquisitions for a period of 12 weeks.

\section{Discussion}

Like many other hospitals, QEHB suffers from serial clonal outbreaks of MDR-Aci, which result from the 
Table 2 Genomic locations and other details of 31 single nucleotide variants (SNVs) detected in the genomes of isolates from the Acinetobacter baumannii pulsotype 27 outbreak in Birmingham, UK, 2011 to 2013

\begin{tabular}{|c|c|c|c|c|c|c|c|}
\hline \multirow[t]{2}{*}{ SNV no. } & \multirow{2}{*}{$\begin{array}{l}\text { Location in reference } \\
\text { assembly }\end{array}$} & \multirow[t]{2}{*}{ Orthologue annotation } & \multicolumn{2}{|c|}{ Amino acid } & \multicolumn{2}{|c|}{ Codon (residue in bold) } & \multirow[t]{2}{*}{ Orthologue } \\
\hline & & & Original & New & Original & New & \\
\hline 1 & 2354692 & $\begin{array}{l}\text { Two-component sensor kinase } \\
\text { transcription regulator protein PmrB }\end{array}$ & Pro & Leu & CCA & CTA & AB57_3172 \\
\hline 2 & 1696968 & Diguanylate cyclase & Lys & STOP & AAA & TAA & AB57_0627 \\
\hline 3 & 219628 & $16 \mathrm{~S}$ rRNA methyltransferase GidB & Arg & Ser & CGT & AGT & AB57_1794 \\
\hline 4 & 2953356 & 3-oxoacyl-ACP reductase & Leu & Trp & $\mathrm{TTG}$ & $\mathrm{TGG}$ & AB57_0871 \\
\hline 5 & 2354857 & $\begin{array}{l}\text { Two-component sensor kinase } \\
\text { transcription regulator protein PmrB }\end{array}$ & Thr & \|e & ACT & $\mathrm{ATT}$ & AB57_3172 \\
\hline 6 & 164435 & Adenylate/guanylate cyclase & Asp & Gly & $\mathrm{GAC}$ & GGC & AB57_1850 \\
\hline 7 & 164513 & Adenylate/guanylate cyclase & Tyr & Phe & $\mathrm{TAT}$ & $\mathrm{TTT}$ & AB57_1850 \\
\hline 8 & 2354642 & $\begin{array}{l}\text { Two-component sensor kinase } \\
\text { transcription regulator protein PmrB }\end{array}$ & Thr & Pro & ACC & $\mathrm{CCC}$ & AB57_3172 \\
\hline 9 & 2568699 & Threonine synthase & Leu & Leu & TTA & CTA & AB57_0327 \\
\hline 10 & 555356 & Catalase/peroxidase HPI & Leu & \|le & TTA & ATA & AB57_0488 \\
\hline 11 & 2961444 & AraC family transcriptional regulator & Val & Val & GTC & GTT & AB57_1179 \\
\hline 12 & 48566 & LysR family transcriptional regulator & Leu & \|e & CTC & ATC & AB57_1964 \\
\hline 13 & 1778342 & Lysine/ornithine N-monooxygenase BasC & Trp & STOP & $\mathrm{TGG}$ & $\mathrm{TAG}$ & A1S_2384 \\
\hline 14 & 1600195 & $\begin{array}{l}\text { Bifunctional cyclohexadienyl } \\
\text { dehydrogenase/ 3-phosphoshikimate } \\
\text { 1-carboxyvinyltransferase }\end{array}$ & Gly & Ser & GGT & AGT & AB57_2630 \\
\hline 15 & 3658279 & Non-coding & \multicolumn{2}{|c|}{$\begin{array}{l}\text { Intergenic } 88 \text { bp from } \\
\text { start of serB }\end{array}$} & & & \\
\hline 16 & 2448345 & Plasmid replicase protein & His & Tyr & CAC & TAC & ACINIS123_A0022 \\
\hline 17 & 706757 & Putative transport protein & Ala & Thr & $\mathbf{G C T}$ & $\mathrm{ACT}$ & ABAYE2100 \\
\hline 18 & 3286974 & ABC transporter ATP-binding protein & Gly & Cys & GGT & TGT & ABAYE2100 \\
\hline 19 & 2501364 & $\begin{array}{l}\text { Regulatory helix-turn-helix protein, lysR } \\
\text { family protein }\end{array}$ & Val & \|e & GTA & ATA & ABBFA_001413 \\
\hline 20 & 2354659 & $\begin{array}{l}\text { Two-component sensor kinase } \\
\text { transcription regulator protein PmrB }\end{array}$ & Arg & Leu & CGC & $\mathrm{CTC}$ & AB57_3172 \\
\hline 21 & 2720233 & Non-coding & \multicolumn{2}{|c|}{$\begin{array}{l}\text { intergenic } 72 \text { bp from } \\
\text { start of } k d s D\end{array}$} & & & \\
\hline 22 & 3818799 & Argininosuccinate synthase & Val & Val & GTT & GTA & AB57_1152 \\
\hline 23 & 727482 & $\begin{array}{l}\text { Oxidoreductase short-chain } \\
\text { dehydrogenase/reductase family }\end{array}$ & Leu & Leu & $\mathrm{CTA}$ & $\mathrm{CTT}$ & AB57_2417 \\
\hline 24 & 2153319 & Diguanylate cyclase/phosphodiesterase & Tyr & Asn & TAC & $\mathrm{AAC}$ & AB57_2291 \\
\hline 25 & 2879522 & Glutathionylspermidine synthase & Asp & Glu & GAT & GAG & HMPREF0022_00853 \\
\hline 26 & 2055876 & $\begin{array}{l}\text { D-ala-D-ala-carboxypeptidase, } \\
\text { penicillin-binding protein }\end{array}$ & Thr & Lys & $A C G$ & $\mathrm{AAG}$ & AB57_2923 \\
\hline 27 & 2698063 & $\begin{array}{l}\text { D-and L-methionine ABC transporter } \\
\text { ATP-binding protein MetN }\end{array}$ & $\operatorname{Arg}$ & Trp & CGG & TGG & AB57_1716 \\
\hline 28 & 1499950 & Peptidase M20D, amidohydrolase & Glu & Gly & $\mathrm{GAA}$ & GGA & AB57_2996 \\
\hline 29 & 396513 & Non-coding & \multicolumn{2}{|c|}{$\begin{array}{l}\text { intergenic } 80 \mathrm{bp} \text { from } \\
\text { start of TetR/AcrR } \\
\text { transcriptional regulators }\end{array}$} & & & \\
\hline 30 & 2371782 & Hypothetical protein & Val & Ala & $\mathrm{GTT}$ & $\mathrm{GCT}$ & ACIN5074_3260 \\
\hline 31 & 1935255 & Hypothetical protein & Arg & His & CGT & CAT & AB57_1009 \\
\hline
\end{tabular}

Orthologue designations are taken from the completed genome of Acinetobacter baumannii AB0057 (GenBank Accession CP001182). Coding sequences in which more than one SNV occurs are highlighted in bold. 


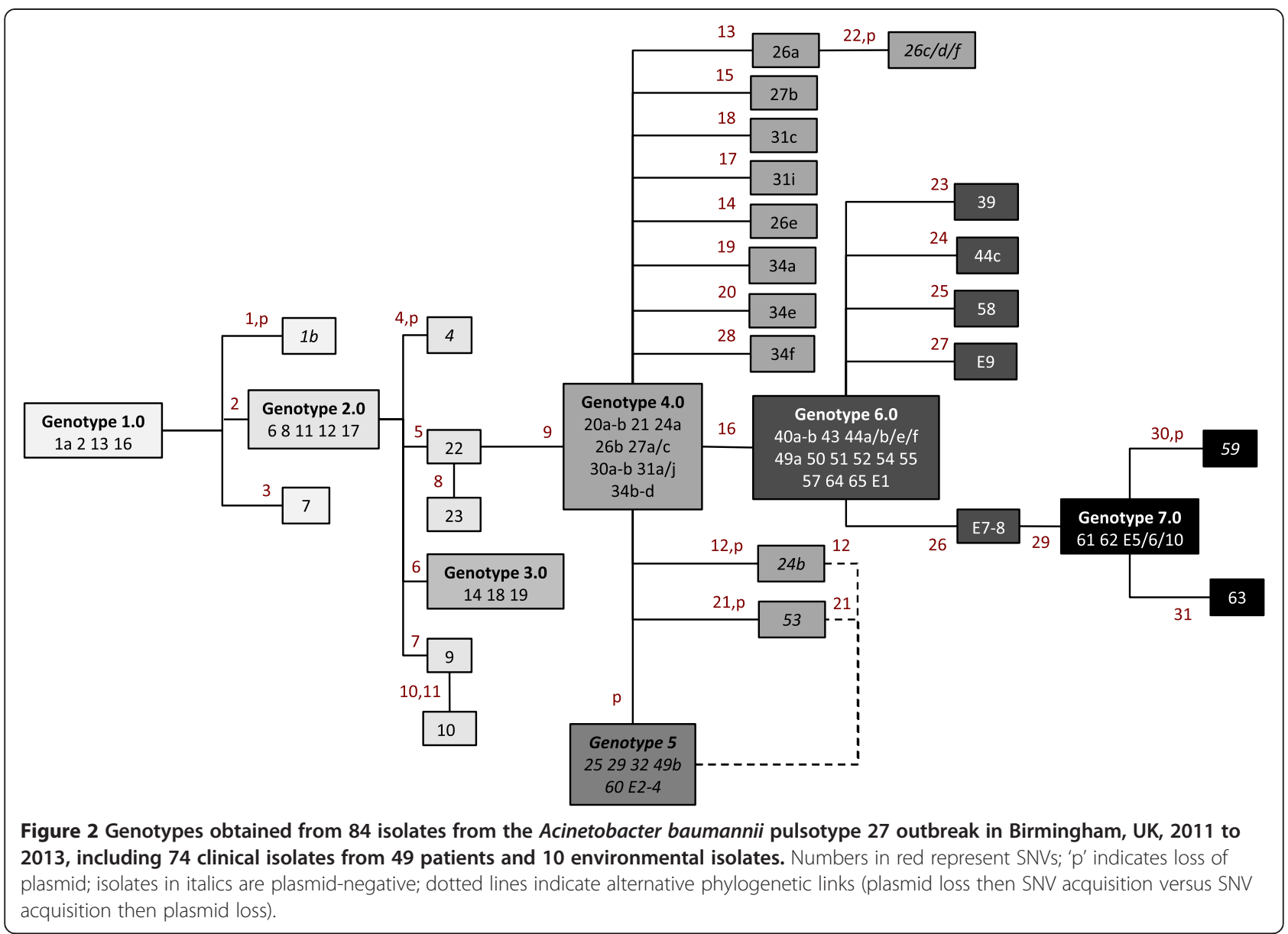

Table 3 Acinetobacter isolates from the Queen Elizabeth Hospital, Birmingham, England cultured between July 2011 and February 2013 that do not belong to Acinetobacter baumannii pulsotype 27

\begin{tabular}{|c|c|c|c|c|c|c|c|}
\hline \multirow[t]{2}{*}{ Patient no. } & \multirow{2}{*}{$\begin{array}{l}\text { Length of hospital } \\
\text { stay (days) }\end{array}$} & \multirow[t]{2}{*}{ Isolate no. } & \multicolumn{2}{|c|}{ Time of isolation (days) } & \multirow[t]{2}{*}{ Species } & \multirow[t]{2}{*}{ Pulsotype } & \multirow[t]{2}{*}{ SNV genotype } \\
\hline & & & After admission & From start of outbreak & & & \\
\hline 3 & 72 & 3 & 21 & 13 & A. baumannii & 3 & Unrelated \\
\hline 5 & 22 & 5 & 2 & 27 & A. baumannii & Unique & Unrelated \\
\hline 26 & 197 & $26 \mathrm{~g}$ & 102 & 265 & A. baumannii & Not typed & Unrelated \\
\hline 37 & 25 & 37 & 3 & 291 & A. baumannii & Not typed & Unrelated \\
\hline 41 & 72 & 41 & 10 & 371 & A. baumannii & 13 & Unrelated \\
\hline 45 & 35 & 45 & 57 & 394 & A. baumannii & Unique & Unrelated \\
\hline 47 & 48 & 47 & 13 & 401 & A. baumannii & 29 & Related \\
\hline 48 & 167 & 48 & 10 & 404 & A. baumannii & 29 & Related \\
\hline \multirow[t]{2}{*}{46} & 33 & $46 a$ & 5 & 396 & A. baumannii & 29 & Related \\
\hline & & $46 b$ & 7 & 398 & A. baumannii & Not typed & Related \\
\hline 56 & 21 & 56 & 18 & 531 & A. baumannii & 9 & Unrelated \\
\hline 33 & 63 & 33 & 9 & 271 & A. pittii & Not typed & Unrelated \\
\hline 35 & 205 & 35 & 1 & 286 & A. pittii & Not typed & Unrelated \\
\hline 36 & 27 & 36 & 1 & 286 & A. pittii & Not typed & Unrelated \\
\hline 42 & 72 & $42 a, b, c$ & 1 & 373 & A. pittii & Not typed & Unrelated \\
\hline 44 & 15 & $44 d$ & 9 & 391 & A. pittii & Not typed & Unrelated \\
\hline
\end{tabular}


Table 4 Potential transmission events within the Acinetobacter baumannii pulsotype 27 outbreak in Birmingham, England, 2011 to 2013, reconstructed using a parsimonious analysis of ward/theatre occupancy and SNV genotype

\begin{tabular}{llll}
\hline $\begin{array}{l}\text { Patient } \\
\text { no. }\end{array}$ & $\begin{array}{l}\text { Predicted donor(s) } \\
\text { of infection }\end{array}$ & $\begin{array}{l}\text { SNVs compared to } \\
\text { predicted donor(s) }\end{array}$ & $\begin{array}{l}\text { Days between donor } \\
\text { and recipient(s) }\end{array}$ \\
\hline 1 & Index case: a military patient repatriated from Afghanistan \\
2 & 1 & 0 & 0 \\
4 & 1 & 3 & 0 \\
6 & 1 & 1 & 0 \\
7 & 1 & 1 & 0 \\
8 & 6 & 0 & 0 \\
9 & Unknown & & \\
10 & 9 & 2 & 0 \\
11 & 6 or 8 & 0,0 & 0 \\
12 & 6 or 8 or 11 & $0,0,0$ & 0 \\
13 & 1 & 0 & $<14$ \\
14 & 6 or 8 or 11 or 12 & $1,1,1,1$ & 0
\end{tabular}

15 Not included in transmission analysis: strain lost on subculture

Not included in transmission analysis: not genome-sequenced 34

34

34

34

43

44

Unknown

Unknown

Outbreak restarts after patient 52 nursed on bed previously used by patient 50
Table 4 Potential transmission events within the Acinetobacter baumannii pulsotype 27 outbreak in Birmingham, England, 2011 to 2013, reconstructed using a parsimonious analysis of ward/theatre occupancy and SNV genotype (Continued)

\begin{tabular}{llll}
\hline 53 & Unknown & & \\
54 & Unknown & & \\
55 & 54 & 0 & Theatre (1 day gap) \\
57 & 50 & 0 & $<21$ \\
58 & 57 & 1 & 0 \\
59 & 57 & 2 & 0 \\
60 & 54 & 1 (loss of plasmid) & $<14$ \\
61 & 57 & 2 & Theatre (4 day gap) \\
62 & 57 & 2 & 0 \\
63 & 62 & 1 & 0 \\
64 & 57 & 0 & $<14$ \\
65 & 64 & 0 & 0 \\
\hline
\end{tabular}

importations of outbreak strains, often by military patients [7-9]. We have described a prolonged outbreak of MDR-Aci, in which bacterial WGS provided a powerful adjunct to conventional laboratory and epidemiological investigations. In so doing, we have built on our previous smaller-scale efforts on the genomic epidemiology of A. baumannii [7] and on the work of others on a polyclonal outbreak genome-sequenced using the 454 platform [28]. However, we have now benefited from the improved ease of use, cost-effectiveness, throughput and accuracy of the Illumina MiSeq platform. Unlike a recent epidemiological analysis of MDR-Aci in a US hospital [29]. We have focused on a single clonal outbreak and have used a whole-genome SNV-based analysis twinned with loss or gain of a plasmid to reconstruct strain evolution as the outbreak progressed.

We have shown that this particular pathogen, Acinetobacter baumannii, undergoes sufficiently rapid genome evolution within a hospital outbreak to allow SNV analyses to reveal modes and routes of transmission and distinguish between alternate transmission scenarios. When we combined evidence from over 30 genotypes with conventional epidemiological data, we were able to identify the sources of several unexplained transmission events and target additional investigations and infection-control interventions to reduce the risk of further transmission.

In this study, we were able to link patient-derived isolates directly to environmental isolates. Early in the outbreak, confirmation of contamination of the patients' surroundings on the ward led to a tightening of ward disinfection procedures. Later, WGS showed that environmental isolates from a burns operating theatre were 
linked to patient isolates, which triggered closure of the theatre, followed by deep cleaning. Previous reports have highlighted that burns patients are particularly at risk of infection with Acinetobacter baumannii and that burns units are susceptible to outbreaks and have also implicated contaminated mattresses in infection [30,31].

We have demonstrated that there is considerable genotypic diversity within patient-associated and environmental populations of Acinetobacter. We discovered two examples of what were clearly mixed infections, where more than one species or strain of Acinetobacter was present in serial samples from the same site. In addition, when we genome-sequenced multiple isolates from multiple samples from a chronically infected patient, we found that the individual was carrying a cloud of variants of the outbreak strain, rather than a single SNV genotype. It remains unclear whether such genotypic diversity evolves in situ or whether multiple genotypes are acquired serially or en masse. Although some have suggested that such genotypic diversity precludes the reconstruction of transmission events from genomic data [32], the congruence and consistency we and others [12] see between genotypic and epidemiologic data in such analyses confirm the utility of such approaches. However, the presence of such genotypic diversity in pathogen populations challenges the long held practice of picking and propagating a single example of each colonial morphotype from a primary culture and suggests a role for metagenomic approaches that might capture bacterial genetic heterogeneity directly from the clinical sample without culture [33-35].

Although, in this study, WGS was initially largely retrospective, in the latter part of the outbreak, we were able to go from colony to SNV genotype in less than 1 week. Local WGS thus allowed us to rule patients and isolates in or out of the outbreak more quickly than through the reference laboratory. Excluding isolates meant that we could focus our efforts on determining the connections between genetically related cases, rather than trying to connect all cases of MDR-Aci.

\section{Conclusions}

WGS is now poised to make an impact on hospital infection prevention and control, delivering cost-effective identification of routes of infection within a clinically relevant timeframe and allowing infection control teams to track, and even prevent, the spread of drug-resistant hospital pathogens.

\section{Abbreviations}

MDR-Aci: Multi-drug-resistant Acinetobacter; MIC: Minimum inhibitory concentration; PFGE: Pulsed field gel electrophoresis; QEHB: Queen Elizabeth Hospital Birmingham; SNV: Single nucleotide variant; WGS: Whole-genome sequencing.
Competing interests

The authors declare that they have no competing interests.

\section{Authors' contributions}

$\mathrm{MRH}$ performed bioinformatics analyses of genomes and wrote a script for assessing the parsimonious transmission routes; JZ-MC performed whole genome sequencing and bioinformatics analysis and helped write the paper; $\mathrm{CIC}$ helped design the study and performed whole genome sequencing; NC, CB and M-SB supplied isolates and patient metadata; BO oversaw clinica investigation of the outbreak, helped design the study and write the manuscript; MJP conceived, designed and supervised the study and wrote the manuscript. All authors read and approved the final manuscript.

\section{Acknowledgements}

We thank Jane Turton and her team at Public Health England for providing strains and access to routinely collected typing information; we thank Nick Loman, Danny Wilson and Mark Achtman for advice in drafting the manuscript. This paper presents independent research funded by the UK Medical Research Council (MRC) and the National Institute for Health Research (NIHR) Surgical Reconstruction and Microbiology Research Centre (partnership between University Hospitals Birmingham NHS Foundation Trust, the University of Birmingham and the Royal Centre for Defence Medicine). The views expressed are those of the author(s) and not necessarily those of the MRC, NHS, the NIHR or the Department of Health.

\section{Author details}

'Institute of Microbiology and Infection, University of Birmingham, Birmingham B15 2TT, UK. ²Division of Microbiology and Infection, Warwick Medical School, University of Warwick, Warwick CV4 7AL, UK. ${ }^{3} \mathrm{NIHR}$ Surgical Reconstruction and Microbiology Research Centre, Queen Elizabeth Medical Centre, Birmingham B15 2TH, UK.

Received: 19 June 2014 Accepted: 8 September 2014

Published online: 20 November 2014

\section{References}

1. Munoz-Price LS, Weinstein RA: Acinetobacter infection. N Engl J Med 2008, 358:1271-1281.

2. Towner KJ: Acinetobacter: an old friend, but a new enemy. $J$ Hosp Infect 2009, 73:355-363.

3. Roca I, Espinal P, Vila-Farres X, Vila J: The Acinetobacter baumannii oxymoron: commensal hospital dweller turned pan-drug-resistant menace. Front Microbiol 2012, 3:148.

4. O'Shea MK: Acinetobacter in modern warfare. Int J Antimicrob Agents 2012, 39:363-375.

5. Whitman TJ, Qasba SS, Timpone JG, Babel BS, Kasper MR, English JF Sanders JW, Hujer KM, Hujer AM, Endimiani A, Eshoo MW, Bonomo RA: Occupational transmission of Acinetobacter baumannii from a United States serviceman wounded in Iraq to a health care worker. Clin Infect Dis 2008, 47:439-443.

6. Hujer KM, Hujer AM, Hulten EA, Bajaksouzian S, Adams JM, Donskey CJ Ecker DJ, Massire C, Eshoo MW, Sampath R, Thomson JM, Rather PN, Craft DW, Fishbain JT, Ewell AJ, Jacobs MR, Paterson DL, Bonomo RA: Analysis of antibiotic resistance genes in multidrug-resistant Acinetobacter $\mathrm{sp}$. isolates from military and civilian patients treated at the Walter Reed Army Medical Center. Antimicrob Agents Chemother 2006, 50:4114-4123.

7. Lewis T, Loman NJ, Bingle L, Jumaa P, Weinstock GM, Mortiboy D, Pallen MJ: High-throughput whole-genome sequencing to dissect the epidemiology of Acinetobacter baumannii isolates from a hospital outbreak. J Hosp Infect 2010, 75:37-41.

8. Jones A, Morgan D, Walsh A, Turton J, Livermore D, Pitt T, Green A, Gill M, Mortiboy D: Importation of multidrug-resistant Acinetobacter spp infections with casualties from Iraq. Lancet Infect Dis 2006, 6:317-318.

9. Turton JF, Kaufmann ME, Gill MJ, Pike R, Scott PT, Fishbain J, Craft D, Deye G, Riddell S, Lindler LE, Pitt TL: Comparison of Acinetobacter baumannii isolates from the United Kingdom and the United States that were associated with repatriated casualties of the Iraq conflict. J Clin Microbiol 2006, 44:2630-2634. 
10. Turton JF, Gabriel SN, Valderrey C, Kaufmann ME, Pitt TL: Use of sequencebased typing and multiplex PCR to identify clonal lineages of outbreak strains of Acinetobacter baumannii. Clin Microbiol Infect 2007, 13:807-815.

11. Turton JF, Baddal B, Perry C: Use of the accessory genome for characterization and typing of Acinetobacter baumannii. J Clin Microbiol 2011, 49:1260-1266.

12. Robinson ER, Walker TM, Pallen MJ: Genomics and outbreak investigation: from sequence to consequence. Genome Med 2013, 5:36.

13. Reuter S, Ellington MJ, Cartwright EJ, Koser CU, Torok ME, Gouliouris T, Harris SR, Brown NM, Holden MT, Quail M, Parkhill J, Smith GP, Bentley SD, Peacock SJ: Rapid bacterial whole-genome sequencing to enhance diagnostic and public health microbiology. JAMA Intern Med 2013, 173:1397-1404.

14. Gardy JL, Johnston JC, Ho Sui SJ, Cook VJ, Shah L, Brodkin E, Rempel S, Moore R, Zhao Y, Holt R, Varhol R, Birol I, Lem M, Sharma MK, Elwood K, Jones SJ, Brinkman FS, Brunham RC, Tang P: Whole-genome sequencing and social-network analysis of a tuberculosis outbreak. N Engl J Med 2011, 364:730-739

15. Snitkin ES, Zelazny AM, Thomas PJ, Stock F, Henderson DK, Palmore TN, Segre JA: Tracking a hospital outbreak of carbapenem-resistant Klebsiella pneumoniae with whole-genome sequencing. Sci Transl Med 2012, 4:148ra116.

16. Eyre DW, Cule ML, Wilson DJ, Griffiths D, Vaughan A, O'Connor L, Ip CL, Golubchik T, Batty EM, Finney JM, Wyllie DH, Didelot X, Piazza P, Bowden R, Dingle KE, Harding RM, Crook DW, Wilcox MH, Peto TE, Walker AS: Diverse sources of $C$. difficile infection identified on whole-genome sequencing. N Engl J Med 2013, 369:1195-1205.

17. Rohde H, Qin J, Cui Y, Li D, Loman NJ, Hentschke M, Chen W, Pu F, Peng Y, Li J, Xi F, Li S, Li Y, Zhang Z, Yang X, Zhao M, Wang P, Guan Y, Cen Z, Zhao X, Christner M, Kobbe R, Loos S, Oh J, Yang L, Danchin A, Gao GF, Song Y, Li Y, Yang H, Wang J, Xu J, Pallen MJ, Wang J, Aepfelbacher M, Yang R: Open-source genomic analysis of Shiga-toxin-producing E. coli O104:H4. N Engl J Med 2011, 365:718-724.

18. Joyanes P, Del Carmen CM, Martinez-Martinez L, Perea EJ: Evaluation of the VITEK 2 system for the identification and susceptibility testing of three species of nonfermenting gram-negative rods frequently isolated from clinical samples. J Clin Microbiol 2001, 39:3247-3253.

19. Miller JR, Koren S, Sutton G: Assembly algorithms for next-generation sequencing data. Genomics 2010, 95:315-327.

20. Goris J, Konstantinidis KT, Klappenbach JA, Coenye T, Vandamme P, Tiedje JM: DNA-DNA hybridization values and their relationship to whole-genome sequence similarities. Int J Syst Evol Microbiol 2007, 57:81-91.

21. Chan JZ, Halachev MR, Loman NJ, Constantinidou C, Pallen MJ: Defining bacterial species in the genomic era: insights from the genus Acinetobacter. BMC Microbiol 2012, 12:302.

22. Langmead B, Salzberg SL: Fast gapped-read alignment with Bowtie 2. Nat Methods 2012, 9:357-359.

23. Li H, Handsaker B, Wysoker A, Fennell T, Ruan J, Homer N, Marth G, Abecasis G, Durbin R: The sequence alignment/map format and SAMtools. Bioinformatics 2009, 25:2078-2079.

24. Jawad A, Seifert $H$, Snelling AM, Heritage J, Hawkey PM: Survival of Acinetobacter baumannii on dry surfaces: comparison of outbreak and sporadic isolates. J Clin Microbiol 1998, 36:1938-1941.

25. Beceiro A, Moreno A, Fernandez N, Vallejo JA, Aranda J, Adler B, Harper M, Boyce JD, Bou G: Biological cost and impact on virulence of different mechanisms of colistin resistance in Acinetobacter baumannii. Antimicrob Agents Chemother 2014, 58:518-526.

26. Koser CU, Holden MT, Ellington MJ, Cartwright EJ, Brown NM, OgilvyStuart AL, Hsu LY, Chewapreecha C, Croucher NJ, Harris SR, Sanders M, Enright MC, Dougan G, Bentley SD, Parkhill J, Fraser LJ, Betley JR, Schulz-Trieglaff OB, Smith GP, Peacock SJ: Rapid whole-genome sequencing for investigation of a neonatal MRSA outbreak. N Engl J Med 2012, 366:2267-2275

27. Young BC, Golubchik T, Batty EM, Fung R, Larner-Svensson H, Votintseva AA Miller RR, Godwin H, Knox K, Everitt RG, labal Z, Rimmer AJ, Cule M, Ip CL, Didelot $X$, Harding RM, Donnelly P, Peto TE, Crook DW, Bowden R, Wilson DJ: Evolutionary dynamics of Staphylococcus aureus during progression from carriage to disease. Proc Natl Acad Sci U S A 2012, 109:4550-4555.

28. Snitkin ES, Zelazny AM, Montero Cl, Stock F, Mijares L, Murray PR, Segre JA: Genome-wide recombination drives diversification of epidemic strains of Acinetobacter baumannii. Proc Natl Acad Sci U S A 2011, 108:13758-13763.

29. Wright MS, Haft DH, Harkins DM, Perez F, Hujer KM, Bajaksouzian S, Benard MF, Jacobs MR, Bonomo RA, Adams MD: New insights into dissemination and variation of the health care-associated pathogen Acinetobacter baumannii from genomic analysis. MBio 2014, 5:e00963-13.

30. Sherertz RJ, Sullivan ML: An outbreak of infections with Acinetobacter calcoaceticus in burn patients: contamination of patients' mattresses. J Infect Dis 1985, 151:252-258.

31. Babik J, Bodnarova L, Sopko K: Acinetobacter- serious danger for burn patients. Acta Chir Plast 2008, 50:27-32.

32. Worby CJ, Lipsitch M, Hanage WP: Within-host bacterial diversity hinders accurate reconstruction of transmission networks from genomic distance data. PLoS Comput Biol 2014, 10:e1003549.

33. Loman NJ, Constantinidou C, Christner M, Rohde H, Chan JZ, Quick J, Weir JC, Quince C, Smith GP, Betley JR, Aepfelbacher M, Pallen MJ: A culture-independent sequence-based metagenomics approach to the investigation of an outbreak of Shiga-toxigenic Escherichia coli 0104:H4. JAMA 2013, 309:1502-1510.

34. Pallen MJ: Diagnostic metagenomics: potential applications to bacterial, viral and parasitic infections. Parasitology 2014, [Epub ahead of print].

35. Schloissnig S, Arumugam M, Sunagawa S, Mitreva M, Tap J, Zhu A, Waller A, Mende DR, Kultima JR, Martin J, Kota K, Sunyaev SR, Weinstock GM, Bork P: Genomic variation landscape of the human gut microbiome. Nature 2013, 493:45-50.

doi:10.1186/s13073-014-0070-x

Cite this article as: Halachev et al:: Genomic epidemiology of a protracted hospital outbreak caused by multidrug-resistant Acinetobacter baumannii in Birmingham, England. Genome Medicine 2014 6:70.

\section{Submit your next manuscript to BioMed Central and take full advantage of:}

- Convenient online submission

- Thorough peer review

- No space constraints or color figure charges

- Immediate publication on acceptance

- Inclusion in PubMed, CAS, Scopus and Google Scholar

- Research which is freely available for redistribution 